\title{
Bose-Einstein condensation and gauge symmetry breaking
}

\author{
V.I. Yukalov \\ Bogolubov Laboratory of Theoretical Physics, \\ Joint Institute for Nuclear Research, Dubna 141980, Russia
}

\begin{abstract}
The fundamental problem is analized, the relation between Bose-Einstein condensation and spontaneous gauge symmetry breaking. This relation is largerly misunderstood in physics community. Numerous articles and books contain the statement that, though gauge symmetry breaking helps for describing Bose-Einstein condensation, but the latter, in principle, does not require any symmetry breaking. This, however, is not correct. The analysis is based on the known mathematical theorems. But in order not to overcomplicate the presentation and to make it accessible to all readers, technical details are often omitted here. The emphasis is made on the following basic general facts: Spontaneous breaking of gauge symmetry is the necessary and sufficient condition for Bose-Einstein condensation. Condensate fluctuations, in thermodynamic limit, are negligible. Their catastrophic behavior can arise only as a result of incorrect calculations, when a Bose-condensed system is described without gauge symmetry breaking. It is crucially important to employ the representative statistical ensembles equipped with all conditions that are necessary for a unique and mathematically correct description of the given statistical system. Only then one is able to develop a self-consistent theory, free of paradoxes.
\end{abstract}

Key words: Bose-Einstein condensation; gauge symmetry breaking; method of infinitesimal sources; Bogolubov operator shift; thermodynamic limit; condensate fluctuations; representative statistical ensembles

PACS: 03.75.Hh, 03.75.Nt, 05.30.Ch, 05.30.Jp, 05.70.Fh, 67.40.Db 


\section{Introduction}

In recent years, the phenomenon of Bose-Einstein condensation (BEC) has received much attention, both experimentally and theoretically (see, e.g., the book [1] and review articles $[2-6])$. At the present time, Bose-Einstein condensates of trapped atoms have been realized in about 70 laboratories of 15 countries, USA, Germany, France, New Zealand, England, Japan, Italy, Netherlands, Israel, Australia, China, Austria, Switzerland, Canada, and Brazil. Magnetic, magneto-optical, and all-optical traps have been employed. BEC has been achieved for 11 atomic species $\left({ }^{1} \mathrm{H},{ }^{4} \mathrm{He},{ }^{7} \mathrm{Li},{ }^{23} \mathrm{Na},{ }^{39} \mathrm{~K},{ }^{41} \mathrm{~K},{ }^{52} \mathrm{Cr},{ }^{85} \mathrm{Rb},{ }^{87} \mathrm{Rb},{ }^{133} \mathrm{Cs}\right.$, and ${ }^{147} \mathrm{Yb}$ ), four types of molecules formed by Bose atoms $\left({ }^{23} \mathrm{Na}_{2},{ }^{85} \mathrm{Rb}_{2},{ }^{87} \mathrm{Rb}_{2}\right.$, and $\left.{ }^{133} \mathrm{Cs}_{2}\right)$, and for two types of molecules formed by Fermi atoms $\left({ }^{6} \mathrm{Li}_{2}\right.$ and $\left.{ }^{40} \mathrm{~K}_{2}\right)$. More details can be found in Refs. [2,5,7-10].

One of course, always remembers that BEC is assumed to exist in superfluid ${ }^{4} \mathrm{He}$, which can be revealed, e.g., in scattering experiments with neutrons $[11-14]$ and $x$-rays $[15,16]$. Also, BEC of boson-type quark clusters might arise in interiors of neutron stars and in heavy-ion collisions [17-19]. There exist several other examples of BEC, which will be cited in the last section of the paper. Thus, Bose-condensed systems form quite a numerous family.

In the theory of Bose-condensed systems, there have remained some principal problems that have not been properly understood in a large part of physics community. One of such fundamental problems is the relation between BEC and the U(1) gauge symmetry breaking. To understand the issue, it is necessary to give answers to the following group of mutually interrelated questions: Is gauge symmetry breaking only a sufficient condition for BEC or it is also a necessary one? In what sense one should interpret the statement that BEC and gauge symmetry breaking are equivalent? What is the difference between the two methods of gauge symmetry breaking, with the help of infinitesimal sources and by means of the Bogolubov shift? What is the origin of the catastrophic behavior of condensate fluctuations in Bose-condensed systems? And what are the requirements for making a theory, with broken gauge symmetry, self-consistent? The aim of the present paper is to answer these questions.

\section{Expansions over natural orbitals}

We shall consider a statistical system of $N$ bosons in volume $V$. For simplicity, we keep in mind the particles with no internal degrees of freedom. After answers to the principal questions are understood, it is easy to generalize the consideration to particles with internal degrees of freedom, when fragmented condensates [20] might arise.

The basic variable, characterizing particles of a statistical system, is the field operator $\psi(\mathbf{r}, t)$, where $\mathbf{r}$ is spatial vector and $t$, time. Bose particles correspond to the Bose commutation relations

$$
\left[\psi(\mathbf{r}, t), \psi^{\dagger}\left(\mathbf{r}^{\prime}, t\right)\right]=\delta\left(\mathbf{r}-\mathbf{r}^{\prime}\right)
$$

with other commutation relations for $[\psi, \psi]$ and $\left[\psi^{\dagger}, \psi^{\dagger}\right]$ being zeros.

\subsection{Expansion of density matrix}

A general way for characterizing BEC is the Penrose-Onsager scheme [21], based on the consideration of the eigenvalues of the first-order density matrix, which is defined as the 
statistical average

$$
\rho\left(\mathbf{r}, \mathbf{r}^{\prime}, t\right) \equiv<\psi^{\dagger}\left(\mathbf{r}^{\prime}, t\right) \psi(\mathbf{r}, t)>.
$$

The eigenvalues $n_{k}$ are given by the eigenproblem

$$
\int \rho\left(\mathbf{r}, \mathbf{r}^{\prime}, t\right) \varphi_{k}\left(\mathbf{r}^{\prime}, t\right) d \mathbf{r}^{\prime}=n_{k}(t) \varphi_{k}(\mathbf{r}, t),
$$

whose eigenfunctions $\varphi_{k}$ are termed natural orbitals [22]. The density matrix (2) acquires the spectral resolution

$$
\rho\left(\mathbf{r}, \mathbf{r}^{\prime}, t\right)=\sum_{k} n_{k}(t) \varphi_{k}(\mathbf{r}, t) \varphi_{k}^{*}\left(\mathbf{r}^{\prime}, t\right)
$$

over the natural orbitals.

For an equilibrium system, the density matrix (2) does not depend on time. Respectively, $n_{k}$ and $\varphi_{k}$ do not depend on time as well, so that the spectral resolution (4) becomes

$$
\rho\left(\mathbf{r}, \mathbf{r}^{\prime}\right)=\sum_{k} n_{k} \varphi_{k}(\mathbf{r}) \varphi_{k}^{*}\left(\mathbf{r}^{\prime}\right) .
$$

In Eqs. (3), (4), and (5), the index $k$ is an appropriate multi-index labelling the eigenvalues and eigenfunctions of eigenproblem (3).

In what follows, we shall mainly deal with the equilibrium case (5), keeping in mind that it can straightforwardly be extended to the general nonequilibrium situation of Eq. (4).

Among the eigenvalues $n_{k}$, let is separate the largest one

$$
N_{0} \equiv \sup _{k} n_{k}
$$

related to a state $\varphi_{0}(\mathbf{r}) \equiv \varphi_{k_{0}}(\mathbf{r})$ labelled by a multi-index $k_{0}$. One says that there occurs BEC, when the largest eigenvalue $N_{0}$ is proportional to the total number of particles $N$, so that $N_{0} \propto N$ for all $N$, including the thermodynamic limit, when

$$
N \rightarrow \infty, \quad V \rightarrow \infty, \quad \frac{N}{V} \rightarrow \text { const },
$$

where a positive constant is implied. Thence, BEC, by definition, is characterized by the condition

$$
\lim _{N \rightarrow \infty} \frac{N_{0}}{N}>0,
$$

where the limit means the thermodynamic limit (7). Under this condition, the spectral resolution (5) can be rewritten as the sum

$$
\rho\left(\mathbf{r}, \mathbf{r}^{\prime}\right)=N_{0} \varphi_{0}(\mathbf{r}) \varphi_{0}^{*}\left(\mathbf{r}^{\prime}\right)+\sum_{k \neq 0} n_{k} \varphi_{k}(\mathbf{r}) \varphi_{k}^{*}\left(\mathbf{r}^{\prime}\right) .
$$

The natural orbitals are assumed to be normalized to one, $\left\|\varphi_{k}\right\|=1$. Therefore, $N_{0}$ is the number of condensed particles.

Often one connects the appearance of BEC with the off-diagonal long-range order, defining the condensate density as the limit

$$
\rho_{0} \equiv \frac{N_{0}}{V}=\lim _{r \rightarrow \infty} \rho(\mathbf{r}, 0) .
$$


This, however, has sense only for an equilibrium uniform system, when $\varphi_{0}=1 / \sqrt{V}$ and the second term in Eq. (9) tends to zero with $r \equiv|\mathbf{r}| \rightarrow \infty$. For example, for a nonuniform confined system, one has $\varphi_{k}(\mathbf{r}) \rightarrow 0$ as $r \rightarrow \infty$, which would mean that for this nonuniform system BEC would be impossible [2].

The Penrose-Onsager criterion of BEC, in the form of condition (8), where $N_{0}$ is the largest eigenvalue (6), is, clearly, more general. Another general form of the BEC criterion can be given by introducing the order indices $[22,23]$. Let us consider $\hat{\rho}_{1} \equiv\left[\rho\left(\mathbf{r}, \mathbf{r}^{\prime}\right)\right]$ as a matrix with respect to the variables $\mathbf{r}$ and $\mathbf{r}^{\prime}$. Then the order index of the matrix is defined $[22,23]$ as

$$
\omega\left(\hat{\rho}_{1}\right) \equiv \frac{\log \left\|\hat{\rho}_{1}\right\|}{\log \operatorname{Tr} \hat{\rho}_{1}}
$$

where $\left\|\hat{\rho}_{1}\right\|$ is the norm of $\hat{\rho}_{1}$. For large $N \gg 1$, this takes the form

$$
\omega\left(\hat{\rho}_{1}\right)=\frac{\log N_{0}}{\log N} .
$$

The order indices (10) or (11) are defined for an arbitrary system and describe different types of ordering:

$$
\begin{array}{cc}
\omega\left(\hat{\rho}_{1}\right)<0 & (\text { no order }), \\
\omega\left(\hat{\rho}_{1}\right)=0 \quad(\text { short }- \text { range order }), \\
0<\omega\left(\hat{\rho}_{1}\right)<1 \quad(\text { mid }- \text { range order }), \\
\left.\omega\left(\hat{\rho}_{1}\right)=1 \quad \text { (long }- \text { range order }\right) .
\end{array}
$$

The long-range order happens in thermodynamic limit, when $N \rightarrow \infty$ and $N_{0} \propto N$. Then, from Eq. (11) it follows that

$$
\lim _{N \rightarrow \infty} \omega\left(\hat{\rho}_{1}\right)=1 .
$$

The criteria (8) and (12) are, of course, absolutely general and can be applied to any system. However, being defined through the density matrix (2), they are not useful without knowing the latter. But the calculation of the density matrix for an interacting system is not a trivial task. A practical calculational procedure is to be developed.

\subsection{Expansion of field operators}

Let us specify how the spectral resolution (9) for the density matrix can be obtained. The field operator $\psi(\mathbf{r})$ can be expanded over the basis of natural orbitals,

$$
\psi(\mathbf{r})=\sum_{k} a_{k} \varphi_{k}(\mathbf{r})
$$

The operators $a_{k}$ in the $k$-representation obey the commutation relations

$$
\left[a_{k}, a_{p}^{\dagger}\right]=\delta_{k p}, \quad\left[a_{k}, a_{p}\right]=0 .
$$

Generally, we do not know which of the orbitals $\varphi_{k}(\mathbf{r})$ corresponds to the condensate. The answer to this question is easy only for the uniform system, when the multi-index $k$ 
translates into the momentum $\mathbf{k}$, so that the condensate is related to $\mathbf{k}=0$. But in the general case, we cannot decide which of the orbitals is that of the condensate. To decide this, we need to know the eigenvalues of the density matrix (2), with the largest eigenvalue pointing at the condensate. So that, actually, we are yet in the vicious circle. Expansion (13), however, is useful for proving some theorems of the condensate existence.

Assume that we are aware which of the natural orbitals $\varphi_{k}(\mathbf{r})$ is related to the condesate, and let us mark it as $\varphi_{0}(\mathbf{r})$. Then we may separate in expansion (13) the condensate field operator

$$
\psi_{0}(\mathbf{r}) \equiv a_{0} \varphi_{0}(\mathbf{r})
$$

from the remaining part associated with the field operator of uncondensed particles,

$$
\psi_{1}(\mathbf{r}) \equiv \sum_{k \neq 0} a_{k} \varphi_{k}(\mathbf{r})
$$

Hence, expansion (13) can be represented as the sum

$$
\psi(\mathbf{r})=\psi_{0}(\mathbf{r})+\psi_{1}(\mathbf{r}) .
$$

The natural orbitals are supposed to be orthonormal,

$$
\int \varphi_{k}^{*}(\mathbf{r}) \varphi_{p}(\mathbf{r}) d \mathbf{r}=\delta_{k p}
$$

From here, the field operators of condensed and uncondensed particles, by construction, are orthogonal,

$$
\int \psi_{0}^{\dagger}(\mathbf{r}) \psi_{1}(\mathbf{r}) d \mathbf{r}=0
$$

Correctly speaking, the operators $\psi_{0}(\mathbf{r})$ and $\psi_{1}(\mathbf{r})$ are not separate independent operators, but they are just two parts of one field operator $\psi(\mathbf{r})$. All of these operators act on the Fock space $\mathcal{F}(\psi)$ generated by the operator $\psi^{\dagger}$. All operators from the algebra of observables, being functionals of $\psi$, are defined on the Fock space $\mathcal{F}(\psi)$. See mathematical details in Ref. $[24]$.

Neither of the operators $\psi_{0}(\mathbf{r})$ or $\psi_{1}(\mathbf{r})$ represents bosons, since their commutation relations are not of Bose statistics. Really, the former operators obey the commutation relation

$$
\left[\psi_{0}(\mathbf{r}), \psi_{0}^{\dagger}\left(\mathbf{r}^{\prime}\right)\right]=\varphi_{0}(\mathbf{r}) \varphi_{0}^{*}\left(\mathbf{r}^{\prime}\right)
$$

while the latter, the relation

$$
\left[\psi_{1}(\mathbf{r}), \psi_{1}^{\dagger}\left(\mathbf{r}^{\prime}\right)\right]=\sum_{k \neq 0} \varphi_{k}(\mathbf{r}) \varphi_{k}^{*}\left(\mathbf{r}^{\prime}\right)
$$

other commutation relations, such as $\left[\psi_{0}, \psi_{0}\right],\left[\psi_{1}, \psi_{1}\right]$, and $\left[\psi_{0}, \psi_{1}\right]$ being zeros. Involving the $\delta$-function expansion

$$
\delta\left(\mathbf{r}-\mathbf{r}^{\prime}\right)=\sum_{k} \varphi_{k}(\mathbf{r}) \varphi_{k}^{*}\left(\mathbf{r}^{\prime}\right)
$$

that is, the completeness of the natural orbital basis $\left\{\varphi_{k}(\mathbf{r})\right\}$, the commutation relation (20) can be represented as

$$
\left[\psi_{1}(\mathbf{r}), \psi_{1}^{\dagger}\left(\mathbf{r}^{\prime}\right)\right]=\delta\left(\mathbf{r}-\mathbf{r}^{\prime}\right)-\left[\psi_{0}(\mathbf{r}), \psi_{0}^{\dagger}\left(\mathbf{r}^{\prime}\right)\right]=\delta\left(\mathbf{r}-\mathbf{r}^{\prime}\right)-\varphi_{0}(\mathbf{r}) \varphi_{0}^{*}\left(\mathbf{r}^{\prime}\right) .
$$


From the latter equation it follows

$$
\int\left[\psi_{1}(\mathbf{r}), \psi_{1}^{\dagger}\left(\mathbf{r}^{\prime}\right)\right] \psi_{0}\left(\mathbf{r}^{\prime}\right) d \mathbf{r}^{\prime}=0
$$

in agreement with the orthogonality condition (18).

The commutation relations (19), (20), and (21) simplify in thermodynamic limit, provided that the condensate natural orbital satisfies the inequality

$$
\left|\varphi_{0}(\mathbf{r})\right|^{2} \leq \frac{\text { const }}{N^{\nu}} \quad(\nu>0) .
$$

For illustration, we may recall that for a uniform system

$$
\varphi_{0}(\mathbf{r})=\frac{1}{\sqrt{V}}=\sqrt{\frac{\rho}{N}}, \quad\left|\varphi_{0}(\mathbf{r})\right|^{2}=\frac{\rho}{N},
$$

hence inequality (22) is valid, with $\nu=1$. Another example is the gas of atoms trapped in a potential $U(\mathbf{r}) \propto r^{n}$. Then [25], inequality (22) is again valid, with $\nu=1+2 / n$. Under condition (22), one has

$$
\lim _{N \rightarrow \infty}\left|\varphi_{0}(\mathbf{r}) \varphi_{0}^{*}\left(\mathbf{r}^{\prime}\right)\right| \leq \lim _{N \rightarrow \infty} \frac{\text { const }}{N^{\nu}}=0
$$

Therefore, in thermodynamic limit, one can formally write down Eq. (19) as

$$
\lim _{N \rightarrow \infty}\left[\psi_{0}(\mathbf{r}), \psi_{0}^{\dagger}\left(\mathbf{r}^{\prime}\right)\right]=0,
$$

and the commutation relation (20) becomes of the Bose kind,

$$
\lim _{N \rightarrow \infty}\left[\psi_{1}(\mathbf{r}), \psi_{1}^{\dagger}\left(\mathbf{r}^{\prime}\right)\right]=\delta\left(\mathbf{r}-\mathbf{r}^{\prime}\right) .
$$

More rigorously, one should consider Eqs. (19) and (21) as equations for distributions, which have sense being integrated with integrable functions from the class of functions such that

$$
\lim _{N \rightarrow \infty}\left|\int_{V} f\left(\mathbf{r}, \mathbf{r}^{\prime}\right) d \mathbf{r} d \mathbf{r}^{\prime}\right|<\infty
$$

On this class of functions, taking into account condition (22), one gets

$$
\begin{gathered}
\lim _{N \rightarrow \infty} \int_{V}\left[\psi_{0}(\mathbf{r}), \psi_{0}^{\dagger}\left(\mathbf{r}^{\prime}\right)\right] f\left(\mathbf{r}, \mathbf{r}^{\prime}\right) d \mathbf{r} d \mathbf{r}^{\prime}=0, \\
\lim _{N \rightarrow \infty} \int_{V}\left[\psi_{1}(\mathbf{r}), \psi_{1}^{\dagger}\left(\mathbf{r}^{\prime}\right)\right] f\left(\mathbf{r}, \mathbf{r}^{\prime}\right) d \mathbf{r} d \mathbf{r}^{\prime}=\lim _{N \rightarrow \infty} \int_{V} \delta\left(\mathbf{r}-\mathbf{r}^{\prime}\right) f\left(\mathbf{r}, \mathbf{r}^{\prime}\right) d \mathbf{r} d \mathbf{r}^{\prime},
\end{gathered}
$$

which explains Eqs. (23) and (24).

Thus, in thermodynamic limit, the condensate variable (15) can be treated as commuting and the operators of uncondensed particles (16) as usual Bose field operators. But for a finite system, one has to deal with the exact commutation relations (19), (20), and (21).

One says that BEC occurs in thermodynamic limit, when the number of condensed particles

$$
N_{0}=\int<\psi_{0}^{\dagger}(\mathbf{r}) \psi_{0}(\mathbf{r})>d \mathbf{r}=<a_{0}^{\dagger} a_{0}>
$$


satisfies the condensation criteria (8) or (12).

Substituting expansion (13), with notations (15), (16), and (17), into the density matrix (2), and assuming the quantum-number conservation condition

$$
<a_{k}^{\dagger} a_{p}>=\delta_{k p}<a_{k}^{\dagger} a_{k}>
$$

we obtain the spectral resolution (9), in which $N_{0}$ is given by Eq. (25), and

$$
n_{k}=<a_{k}^{\dagger} a_{k}>\text {. }
$$

Nowhere throughout this section, gauge symmetry breaking has been either invoked or even mentioned. The spectral resolution (9) formally does not require the breaking of symmetry [26]. The BEC criteria (8) and (12) also are formulated without involving the notion of symmetry breaking. Because of this, one often states that BEC does not necessarily imply gauge symmetry breaking and that the overall phenomenon of BEC can perfectly be described without breaking the gauge symmetry. The latter conclusion, however, is not correct, since it does not follow from the fact that the spectral resolution (9) and the BEC criteria (8) and (12) can be formulated not mentioning symmetry breaking. Strictly specking, Eqs. (9), (8), and (12) tell us nothing about whether gauge symmetry must be broken or not. To understand this, it is necessary to know the correct way of calculating the density matrix and all other averages.

\section{Methods of symmetry breaking}

For the practical purpose of describing BEC, one usually breaks gauge symmetry. There are two ways of doing this, by the method of infinitesimal sources and by the Bogolubov operator shift.

\subsection{Method of infinitesimal sources}

Let the system Hamiltonian $H[\psi]$, being a functional of the field operator $\psi$, be invariant under the gauge transformation $\psi \rightarrow e^{i \alpha} \psi$, where $\alpha$ is a real number, so that

$$
H\left[e^{i \alpha} \psi\right]=H[\psi] .
$$

To break the gauge symmetry, we can add to the Hamiltonian $H[\psi]$ a term explicitly breaking the symmetry, for instance, defining

$$
H_{\varepsilon}[\psi] \equiv H[\psi]+\varepsilon \sqrt{\rho} \int\left[\psi_{0}^{\dagger}(\mathbf{r})+\psi_{0}(\mathbf{r})\right] d \mathbf{r}
$$

Statistical averages for an equilibrium system are then averaged with the statistical operator

$$
\hat{\rho}_{\varepsilon} \equiv \frac{\exp \left\{-\beta H_{\varepsilon}[\psi]\right\}}{\operatorname{Tr} \exp \left\{-\beta H_{\varepsilon}[\psi]\right\}},
$$

in which $\beta \equiv 1 / T$ is inverse tepmerature. Thus, the statistical average of an operator $\hat{A}$ is given by

$$
<\hat{A}>_{\varepsilon} \equiv \operatorname{Tr} \hat{\rho}_{\varepsilon} \hat{A},
$$


where the trace is over the Fock space $\mathcal{F}(\psi)$.

Since Hamiltonian (27) is not invariant under the gauge transformation, the system symmetry is broken. The manifestation of the broken gauge symmetry is

$$
<\psi(\mathbf{r})>_{\varepsilon} \neq 0
$$

But it is important to find out what happens if the symmetry breaking term is removed. If it is removed before thermodynamic limit, then evidently

$$
\lim _{\varepsilon \rightarrow 0}<\psi(\mathbf{r})>_{\varepsilon}=0
$$

However, the average of $\psi$ may remain nonzero, if the symmetry breaking term is removed after thermodynamic limit. One says that gauge symmetry is spontaneously broken, when

$$
\lim _{\varepsilon \rightarrow 0} \lim _{N \rightarrow \infty}<\psi(\mathbf{r})>_{\varepsilon} \neq 0
$$

For a nonequilibrium system, the statistical operator (28) can be treated as the initial form of the time-dependent statistical operator $\hat{\rho}_{\varepsilon}(t)$, so that $\hat{\rho}_{\varepsilon}(0)=\hat{\rho}_{\varepsilon}$.

The averages of type (32), defined through the double noncommuting limiting procedure $\lim _{\varepsilon \rightarrow 0} \lim _{N \rightarrow \infty}\langle\ldots\rangle$, are the Bogolubov quasiaverages $[27,28]$. It is also possible to define the thermodynamic quasiaverages [29-31] involving the sole limiting procedure, that of thermodynamic limit $\lim _{N \rightarrow \infty}<\ldots>$. The latter procedure can also result in the spontaneous symmetry breaking, provided the infinitesimal source in thermodynamic limit tends to zero in the appropriate way [29-31].

\subsection{Bogolubov operator shift}

Another method of gauge symmetry breaking is based on the observation that in thermodynamic limit we come to the commutation relations (23) and (24). The first tells us that the operator $\psi_{0}(\mathbf{r})$ is asymptotically equivalent to a nonoperator function, which can be expressed as the replacement

$$
\psi_{0}(\mathbf{r}) \rightarrow \eta(\mathbf{r}), \quad a_{0} \rightarrow \sqrt{N_{0}}
$$

where $\eta(\mathbf{r})$ is termed the condensate wave function. Respectively, the field operator (17) is to be replaced as

$$
\psi(\mathbf{r}) \rightarrow \hat{\psi}(\mathbf{r}) \equiv \eta(\mathbf{r})+\psi_{1}(\mathbf{r}),
$$

which is called the Bogolubov operator shift. The commutation relation (24) signifies that the operator $\psi_{1}(\mathbf{r})$ asymptotically acquires the usual Bose commutation relations. This agrees with the fact that if $\hat{\psi}(\mathbf{r})$ is a Bose operator and $\eta(\mathbf{r})$ is a nonoperator function, then $\psi_{1}(\mathbf{r})$ in Eq. (34) has to be a Bose operator with the commutation relation

$$
\left[\psi_{1}(\mathbf{r}), \psi_{1}^{\dagger}\left(\mathbf{r}^{\prime}\right)\right]=\delta\left(\mathbf{r}-\mathbf{r}^{\prime}\right)
$$

The orthogonality condition (18) transforms into

$$
\int \eta^{*}(\mathbf{r}) \psi_{1}(\mathbf{r}) d \mathbf{r}=0
$$


Thus, after the Bogolubov shift (34), instead of one field operator $\psi(\mathbf{r})$, as in Eq. (17), there appear two independent variables, the condensate wave function $\eta(\mathbf{r})$ and the field operator of uncondensed particles $\psi_{1}(\mathbf{r})$, satisfying the Bose commutation relations (35).

The field operator $\psi_{1}^{\dagger}$ generates the Fock space $\mathcal{F}\left(\psi_{1}\right)$, which is orthogonal to the Fock space $\mathcal{F}(\psi)$ generated by the operator $\psi^{\dagger}$. The Bogolubov shift (34) realizes the unitary nonequivalent operator representations [32]. Consequently, any operator $\hat{A}[\psi]$, expressed through the field operator $\psi$, is defined on the space $\mathcal{F}(\psi)$, while the corresponding operator $\hat{A}\left[\eta, \psi_{1}\right]$ acts on the space $\mathcal{F}\left(\psi_{1}\right)$.

Accomplishing the Bogolubov shift (34), we pass from the Hamiltonian $H[\psi]$ to the Hamiltonian $H\left[\eta, \psi_{1}\right]$. Then the average

$$
<\hat{A}\left[\eta, \psi_{1}\right]>_{\eta} \equiv \operatorname{Tr} \hat{\rho}(\eta) \hat{A}\left[\eta, \psi_{1}\right]
$$

is defined as the trace over $\mathcal{F}\left(\psi_{1}\right)$, with the statistical operator

$$
\hat{\rho}(\eta) \equiv \frac{\exp \left\{-\beta H\left[\eta, \psi_{1}\right]\right\}}{\operatorname{Tr} \exp \left\{-\beta H\left[\eta, \psi_{1}\right]\right\}} .
$$

Using average (37), one introduces the quantum-number conservation condition

$$
<\psi_{1}(\mathbf{r})>_{\eta}=0
$$

which defines the operator average

$$
<\hat{\psi}(\mathbf{r})>_{\eta}=\eta(\mathbf{r})
$$

as the order parameter of the system. The condensate wave function is normalized to the number of condensed particles

$$
N_{0}=\int|\eta(\mathbf{r})|^{2} d \mathbf{r}
$$

If the system is absolutely stable and the number $N_{0}$ satisfies the condensation criteria (8) or (12), then there exists BEC.

The Hamiltonian (27), with the infinitesimal term, transforms to $H_{\varepsilon}\left[\eta, \psi_{1}\right]$. The related statistical operator becomes

$$
\hat{\rho}_{\varepsilon}(\eta) \equiv \frac{\exp \left\{-\beta H_{\varepsilon}\left[\eta, \psi_{1}\right]\right\}}{\operatorname{Tr} \exp \left\{-\beta H_{\varepsilon}\left[\eta, \psi_{1}\right]\right\}},
$$

where the trace is over $\mathcal{F}\left(\psi_{1}\right)$. Similarly to Eq. (37), we now have the average

$$
<\hat{A}\left[\eta, \psi_{1}\right]>_{\varepsilon \eta} \equiv \operatorname{Tr} \hat{\rho}_{\varepsilon}(\eta) \hat{A}\left[\eta, \psi_{1}\right] .
$$

Since

$$
\lim _{\varepsilon \rightarrow 0} H_{\varepsilon}\left[\eta, \psi_{1}\right]=H\left[\eta, \psi_{1}\right]
$$

averages (37) and (43) are connected through the relation

$$
\lim _{\varepsilon \rightarrow 0}<\hat{A}\left[\eta, \psi_{1}\right]>_{\varepsilon \eta}=<\hat{A}\left[\eta, \psi_{1}\right]>_{\eta} .
$$


The Bogolubov shift (34), because of condition (40), explicitly breaks gauge symmetry. When the infinitesimal term is added, then $\left\langle\psi_{1}(\mathbf{r})\right\rangle_{\varepsilon \eta}$ is not zero. However, in compliance with Eq. (44), we have

$$
\lim _{\varepsilon \rightarrow 0}<\psi_{1}(\mathbf{r})>_{\varepsilon \eta}=<\psi_{1}(\mathbf{r})>_{\eta}=0 .
$$

Therefore

$$
\lim _{\varepsilon \rightarrow 0}<\hat{\psi}(\mathbf{r})>_{\varepsilon \eta}=<\hat{\psi}(\mathbf{r})>_{\eta}=\eta(\mathbf{r}),
$$

which means that gauge symmetry is broken, provided $\eta(\mathbf{r})$ is not zero. Thus, the Bogolubov shift (34) breaks the gauge symmetry without introducing infinitesimal sources.

\section{Sufficient and necessary conditions}

All observable and thermodynamic quantities of a statistical system can be expressed through some correlation functions. Let us consider the class of correlation functions

$$
C_{\varepsilon}\left(\psi_{0}, \psi_{1}\right) \equiv<\ldots \psi_{0}^{\dagger} \ldots \psi_{1}^{\dagger} \ldots \psi_{0} \ldots \psi_{1} \ldots>_{\varepsilon}
$$

consisting of the averages (29) of a product of the field operators $\psi_{0}^{\dagger}, \psi_{1}^{\dagger}, \psi_{0}$, and $\psi_{1}$ in any order. And let us introduce the class of correlation functions

$$
C\left(\eta, \psi_{1}\right) \equiv<\ldots \eta^{*} \ldots \psi_{1}^{\dagger} \ldots \eta \ldots \psi_{1}>_{\eta}
$$

represented by averages (37). The order of the factors here is the same as in Eq. (46) with the change of $\psi_{0}$ by $\eta$. Also, we define the class of correlation functions

$$
C_{\varepsilon}\left(\eta, \psi_{1}\right) \equiv<\ldots \eta^{*} \ldots \psi_{1}^{\dagger} \ldots \eta \ldots \psi_{1}>_{\varepsilon \eta}
$$

given by the averages (43) of a product of $\eta^{*}, \psi_{1}^{\dagger}, \eta$, and $\psi_{1}$, whose order is the same as in Eq. (47).

The class of correlation functions (46) is defined for a system, where gauge symmetry is broken by means of the infinitesimal source. In this method, the limit $\varepsilon \rightarrow 0$ and the thermodynamic limit do not commute, so that

$$
\left[\lim _{\varepsilon \rightarrow 0}, \lim _{N \rightarrow \infty}\right] C_{\varepsilon}\left(\psi_{0}, \psi_{1}\right) \neq 0 .
$$

The class of correlation functions (48) is constructed by employing the Bogolubov shift (34). In the latter method, gauge symmetry is broken explicitly, because of which the limits $\varepsilon \rightarrow 0$ and $N \rightarrow \infty$ now commute,

$$
\left[\lim _{\varepsilon \rightarrow 0}, \lim _{N \rightarrow \infty}\right] C_{\varepsilon}\left(\eta, \psi_{1}\right)=0 .
$$

\subsection{Sufficient condition for condensation}

Bogolubov $[28,33,34]$ showed that the methods of breaking gauge symmetry by means of infinitesimal sources and by the operator shift (34) are asymptotically equivalent and the operator shift (34) is asymptotically exact in the following sense. Let us compare the classes defined in Eqs. (46), (47), and (48). The correlation functions from these classes will be called similar if they have the same operator structure up to the change of $\psi_{0}$ by $\eta$. 


\section{Bogolubov theorem.}

Similar correlation functions from the classes (46) and (48), in thermodynamic limit, coincide,

$$
\lim _{N \rightarrow \infty} C_{\varepsilon}\left(\psi_{0}, \psi_{1}\right)=\lim _{N \rightarrow \infty} C_{\varepsilon}\left(\eta, \psi_{1}\right) .
$$

This limiting equality holds for any $\varepsilon$, including $\varepsilon \rightarrow 0$. Therefore, taking into account the commutation property (50), one has

$$
\lim _{\varepsilon \rightarrow 0} \lim _{N \rightarrow \infty} C_{\varepsilon}\left(\psi_{0}, \psi_{1}\right)=\lim _{N \rightarrow \infty} C\left(\eta, \psi_{1}\right) .
$$

The limits in the left-hand side of Eq. (52) do not commute.

From the Bogolubov theorem, it follows, in particular, that

$$
\lim _{N \rightarrow \infty}\left|<\psi_{0}(\mathbf{r})>_{\varepsilon}\right|^{2}=\lim _{N \rightarrow \infty}<\psi_{0}^{\dagger}(\mathbf{r}) \psi_{0}(\mathbf{r})>_{\varepsilon} .
$$

This is evident, since Eq. (53), with property (51), reduces to the trivial identity $|\eta|^{2}=|\eta|^{2}$. For a uniform system, Eq. (53) becomes

$$
\lim _{V \rightarrow \infty} \frac{1}{V}\left|<a_{0}>_{\varepsilon}\right|^{2}=\lim _{V \rightarrow \infty} \frac{1}{V}<a_{0}^{\dagger} a_{0}>_{\varepsilon} .
$$

This again immediately follows from the Bogolubov theorem (51) and, according to Eq. (33), reduces to the identity $\rho_{0}=\rho_{0}$. Equality (54) has also been obtained by Belyaev [35] and Ginibre [36] and recently an elegant proof was given by Lieb et al. [37-39].

Equalities (53) and (54) are valid for any $\varepsilon$, including $\varepsilon \rightarrow 0$. Defining the local condensate density

$$
\rho_{0}(\mathbf{r}) \equiv \lim _{\varepsilon \rightarrow 0} \lim _{N \rightarrow \infty}<\psi_{0}^{\dagger}(\mathbf{r}) \psi_{0}(\mathbf{r})>_{\varepsilon}
$$

we find from Eq. (53)

$$
\lim _{\varepsilon \rightarrow 0} \lim _{N \rightarrow \infty}\left|<\psi_{0}(\mathbf{r})>_{\varepsilon}\right|^{2}=\rho_{0}(\mathbf{r}) .
$$

The latter equation shows us that if gauge symmetry is locally spontaneously broken, so that the left-hand side of Eq. (56) is not identically zero, then there exists local BEC in the sense that $\rho_{0}(\mathbf{r})>0$ at least for some $\mathbf{r}$. And if there is no spontaneous symmetry breaking, at least locally, so that the left-hand side of Eq. (56) is identically zero, then there is no $\mathrm{BEC}$, since $\rho_{0}(\mathbf{r})=0$. When spontaneous breaking of gauge symmetry occurs globally, in the sense that

$$
\lim _{\varepsilon \rightarrow 0} \lim _{V \rightarrow \infty} \frac{1}{V} \int_{V}\left|<\psi_{0}(\mathbf{r})>_{\varepsilon}\right|^{2} d \mathbf{r}>0,
$$

then there exists BEC in the usual sense of criteria (8) or (12), since then

$$
\lim _{V \rightarrow \infty} \frac{1}{V} \int_{V} \rho_{0}(\mathbf{r}) d \mathbf{r}>0
$$

For a uniform system, as is clear, the local and global BEC conditions are equivalent.

Note that breaking of gauge symmetry can interchangeably be considered for the averages of either $\psi$ or $\psi_{0}$, since $\psi=\psi_{0}+\psi_{1}$ and from the Bogolubov theorem we have

$$
\lim _{\varepsilon \rightarrow 0} \lim _{N \rightarrow \infty}<\psi_{1}(\mathbf{r})>_{\varepsilon}=\lim _{\varepsilon \rightarrow 0} \lim _{N \rightarrow \infty}<\psi_{1}(\mathbf{r})>_{\varepsilon \eta}=\lim _{N \rightarrow \infty}<\psi_{1}(\mathbf{r})>_{\eta}=0,
$$


where condition (39) is also taken into account. Therefore

$$
\lim _{\varepsilon \rightarrow 0} \lim _{N \rightarrow \infty}<\psi(\mathbf{r})>_{\varepsilon}=\lim _{\varepsilon \rightarrow 0} \lim _{N \rightarrow \infty}<\psi_{0}(\mathbf{r})>_{\varepsilon} .
$$

The asymptotic exactness of the Bogolubov shift (34) has also been proved for thermodynamic potentials by Ginibre [36] and Lieb et al. [37-39]. Let us consider the thermodynamic potentials

$$
\Omega_{\varepsilon} \equiv-T \ln \operatorname{Tr} \exp \left\{-\beta H_{\varepsilon}[\psi]\right\},
$$

with the trace over $\mathcal{F}(\psi)$, and

$$
\Omega_{\varepsilon}(\eta) \equiv-T \ln \operatorname{Tr} \exp \left\{-\beta H_{\varepsilon}\left[\eta, \psi_{1}\right]\right\}
$$

with the trace over $\mathcal{F}\left(\psi_{1}\right)$. And let $\eta$ be the minimizer for the potential

$$
\Omega_{\varepsilon}(\eta)=\inf _{x} \Omega_{\varepsilon}(x)
$$

Then, for sufficiently general conditions on the interaction potential (see details in Refs. [36-39]), one has the Ginibre theorem

$$
\lim _{V \rightarrow \infty} \frac{1}{V}\left[\Omega_{\varepsilon}(\eta)-\Omega_{\varepsilon}\right]=0
$$

Ginibre [36] proved this theorem for $\varepsilon>0$ as well as for $\varepsilon=0$.

The Bogolubov and Ginibre theorems show that two ways of breaking gauge symmetry, by means of infinitesimal sources and by the Bogolubov shift (34), are asymptotically equivalent and that the Bogolubov shift (34) is asymptotically exact in the sense of Eqs. (51), (52), and (61). It also follows that the spontaneous gauge symmetry breaking is a sufficient condition for the existence of $B E C$.

\subsection{Necessary condition for condensation}

The fundamental question is whether the gauge symmetry breaking would also be the necessary condition for BEC. This problem has been discussed in a number of works, of which we mention here just a few $[1,2,22,26,40]$. One often states that gauge symmetry breaking is not necessary for BEC and that the letter can occur without any symmetry breaking. This, however, is a delusion, though a widespread one.

Suppose, we do not break gauge symmetry and work with the statistical operator

$$
\hat{\rho} \equiv \frac{\exp \{-\beta H[\psi]\}}{\operatorname{Tr} \exp \{-\beta H[\psi]\}},
$$

in which $H[\psi]$ is a gauge-symmetric Hamiltonian. The related gauge-symmetric averages are defined as

$$
<\hat{A}>\equiv \operatorname{Tr} \hat{\rho} \hat{A}
$$

For a uniform Bose-condensed system, Roepstorff [41] proved the inequality

$$
\lim _{N \rightarrow \infty} \frac{<a_{0}^{\dagger} a_{0}>}{N} \leq \lim _{\varepsilon \rightarrow 0} \lim _{N \rightarrow \infty} \frac{\left|<a_{0}>_{\varepsilon}\right|^{2}}{N}
$$


In his proof, he used the assumption that, in thermodynamic limit, $H[\psi]$ commutes with $\psi_{0}$. This is a plausible assumption, since, as is discussed above, in thermodynamic limit, $\psi_{0}$ can be replaced by a nonoperator function $\eta$. A more general proof of inequality (64) was given by Lieb et al. in a series of papers [37-39]. Actually, the main requirement, used by Lieb et al. [37-39], was the existence of the Fourier transform for the interaction potential. The case of hard-core potentials can also be taken care of by cutting off the potential at some finite value that is taken to infinity at the end of the calculations [37-39].

In the coordinate representation, the Roepstorff inequality (64) can be written as

$$
\lim _{V \rightarrow \infty} \frac{1}{V} \int_{V}<\psi_{0}^{\dagger}(\mathbf{r}) \psi_{0}(\mathbf{r})>d \mathbf{r} \leq \lim _{\varepsilon \rightarrow 0} \lim _{V \rightarrow \infty} \frac{1}{V} \int_{V}\left|<\psi_{0}(\mathbf{r})>_{\varepsilon}\right|^{2} d \mathbf{r} .
$$

When the left-hand sides of Eqs. (64) or (65) are nonzero, this means the existence of BEC, in compliance with the BEC criteria (8) or (12). But then the right-hand sides of Eqs. (64) and (65) are also nonzero, which implies spontaneous symmetry breaking. That is, the existence of BEC necessarily assumes spontaneous gauge symmetry breaking.

Equalities (64) and (65) are the direct analogs of the Griffiths [42] inequality

$$
\lim _{N \rightarrow \infty}<\hat{\mathbf{S}}^{2}>\leq \lim _{\varepsilon \rightarrow 0} \lim _{N \rightarrow \infty}\left|<\hat{\mathbf{S}}>_{\varepsilon}\right|^{2}
$$

for magnetic systems, where

$$
\hat{\mathbf{S}} \equiv \frac{1}{N} \sum_{i=1}^{N} \hat{\mathbf{S}}_{i}
$$

is the mean spin operator and the symmetry breaking term is $\varepsilon N \hat{\mathbf{S}}^{z}$, so that $\varepsilon$ plays the role of an external magnetic field. The Griffiths inequality tells us that the appearance of magnetization necessarily implies the spontaneously broken spin-rotational symmetry $O(3)$.

In this way, from the Bogolubov theorem, formulated in Eq. (51), equalities (53) to (56) follow, which have also been proved by Ginibre [36] and by Lieb et al. [37-39]. These equalities show that the spontaneous gauge symmetry breaking is a sufficient condition for the occurrence of BEC. And inequalities (64) and (65), proved by Roepstorff [41] and by Lieb et al. [37-39], establish that the existence of BEC is necessarily accompanied by the spontaneous gauge symmetry breaking. Thence, we come to the conclusion:

The spontaneous gauge symmetry breaking is the necessary and sufficient condition for the existence of Bose-Einstein condensate.

\subsection{Illustration by mean-field model}

In order to illustrate the above conclusion explicitly, let us consider a simple mean-field type model with the Hamiltonian

$$
H=\sum_{k}\left(\omega_{k}-\mu\right) a_{k}^{\dagger} a_{k}
$$

where $a_{k}$ and $a_{k}^{\dagger}$ are Bose operators and $\omega_{k}$ is an effective spectrum, such that $\omega_{k}-\mu \geq 0$ for all multi-indices $k$. For a uniform system, the index $k$ becomes the momentum $\mathbf{k}$. But, in general, the system can be of any nature. 
For temperatures $T$ above the condensation temperature $T_{c}$, there is no need to break gauge symmetry. Then the particle distribution is

$$
<a_{k}^{\dagger} a_{k}>=\left[e^{\beta\left(\omega_{k}-\mu\right)}-1\right]^{-1}
$$

for any $k$. The appearance of a Bose condensate presupposes that the ground-state level $\omega_{0}$ gets macroscopically populated, as a result of which the number of particles on this level,

$$
<a_{0}^{\dagger} a_{0}>=\left[e^{\beta\left(\omega_{0}-\mu\right)}-1\right]^{-1}
$$

becomes proportional to the total number of particles $N$. In order that $<a_{0}^{\dagger} a_{0}>\propto N$, it is necessary that

$$
\lim _{N \rightarrow \infty}\left(\omega_{0}-\mu\right) \rightarrow+0 \quad\left(T \leq T_{c}\right) .
$$

Hence, Eq. (69) is a necessary condition for BEC.

Breaking gauge symmetry by the infinitesimal term, as in Eq. (27), we now have

$$
H_{\varepsilon} \equiv H+\varepsilon \sqrt{N}\left(a_{0}^{\dagger}+a_{0}\right)
$$

Hamiltonian (70), with $H$ from Eq. (66), can be diagonalized by means of the canonical transformation

$$
a_{0}=b_{0}+\frac{\varepsilon \sqrt{N}}{\mu-\omega_{0}} .
$$

Then Eq. (70) transforms into

$$
H_{\varepsilon}=\left(\omega_{0}-\mu\right) b_{0}^{\dagger} b_{0}+\sum_{k \neq 0}\left(\omega_{k}-\mu\right) a_{k}^{\dagger} a_{k}+\frac{\varepsilon^{2} N}{\mu-\omega_{0}} .
$$

The latter Hamiltonian is gauge-invariant with respect to the change $b_{0} \rightarrow b_{0} e^{i \alpha}$ and $a_{k} \rightarrow$ $a_{k} e^{i \alpha}$. Therefore,

$$
<b_{0}>_{\varepsilon}=0,
$$

where the averaging is with Hamiltonian (72) and, similarly,

$$
<a_{k}>_{\varepsilon}=0 \quad(k \neq 0) .
$$

From Eq. (71), we find

$$
<a_{0}>_{\varepsilon}=\frac{\varepsilon \sqrt{N}}{\mu-\omega_{0}} .
$$

For the bilinear averages, we get

$$
<b_{0}^{\dagger} b_{0}>_{\varepsilon}=<a_{0}^{\dagger} a_{0}>,
$$

whose form is given by Eq. (68), and

$$
<a_{k}^{\dagger} a_{k}>_{\varepsilon}=<a_{k}^{\dagger} a_{k}>\quad(k \neq 0),
$$

defined by expression (67). 
If there is no BEC, hence, condition (69) does not hold, then there is no spontaneous gauge symmetry breaking, since

$$
\lim _{\varepsilon \rightarrow 0} \lim _{N \rightarrow \infty} \frac{<a_{0}>_{\varepsilon}}{\sqrt{N}}=0 \quad\left(\mu \neq \omega_{0}\right) .
$$

The number of condensed particles is, generally, different being calculated using the averages with gauge symmetry breaking or without it. Let us denote the former as

$$
N_{0}(\varepsilon) \equiv<a_{0}^{\dagger} a_{0}>_{\varepsilon} \quad(\varepsilon \neq 0)
$$

and the latter as

$$
N_{0}(0) \equiv<a_{0}^{\dagger} a_{0}>\quad(\varepsilon \equiv 0) .
$$

In agreement with transformation (71), we have

$$
N_{0}(\varepsilon)=<b_{0}^{\dagger} b_{0}>_{\varepsilon}+\frac{\varepsilon^{2} N}{\left(\mu-\omega_{0}\right)^{2}} .
$$

From here, keeping in mind equality (76), we get

$$
<a_{0}^{\dagger} a_{0}>=N_{0}(0) \leq N_{0}(\varepsilon)=<a_{0}^{\dagger} a_{0}>_{\varepsilon} .
$$

Let below $T_{c}$ the condensate arise, so that $N_{0}(\varepsilon)>0$ and condition (69) holds. Then, for large $N$, from Eq. (81), we obtain

$$
\mu \simeq \omega_{0}-\frac{T}{2 N_{0}(\varepsilon)}\left[1+\sqrt{1+(2 \beta \varepsilon)^{2} N_{0}(\varepsilon) N}\right] .
$$

For $\varepsilon \neq 0$ and $N \rightarrow \infty$, Eq. (83) simplifies to

$$
\mu \simeq \omega_{0}-\varepsilon \sqrt{\frac{N}{N_{0}(\varepsilon)}} .
$$

Using this in transformation (71), we have

$$
a_{0} \simeq b_{0}-\sqrt{N_{0}(\varepsilon)} .
$$

Then, in view of Eq. (73), we obtain

$$
<a_{0}>_{\varepsilon} \simeq-\sqrt{N_{0}(\varepsilon)} .
$$

Equation (85) is analogous to the Bogolubov shift, and Eq. (86) yields

$$
\lim _{N \rightarrow \infty} \frac{\left|<a_{0}>_{\varepsilon}\right|^{2}}{N}=\lim _{N \rightarrow \infty} \frac{N_{0}(\varepsilon)}{N} .
$$

The latter equality, together with definition (79), takes the form

$$
\lim _{N \rightarrow \infty} \frac{\left|<a_{0}>_{\varepsilon}\right|^{2}}{N}=\lim _{N \rightarrow \infty} \frac{<a_{0}^{\dagger} a_{0}>_{\varepsilon}}{N},
$$


which is equivalent to the Ginibre-Lieb equality (54). Equation (88) tells us that asymptotically

$$
<a_{0}^{\dagger} a_{0}>_{\varepsilon} \simeq\left|<a_{0}>_{\varepsilon}\right|^{2} \quad(N \rightarrow \infty) .
$$

Taking in Eq. (88) the limit $\varepsilon \rightarrow 0$, we see that the spontaneous gauge symmetry breaking implies the existence of BEC, being a sufficient condition for the latter.

On the other hand, from Eqs. (82) and (88), with definitions (79) and (80), we obtain

$$
\lim _{N \rightarrow \infty} \frac{<a_{0}^{\dagger} a_{0}>}{N} \leq \lim _{N \rightarrow \infty} \frac{\left|<a_{0}>_{\varepsilon}\right|^{2}}{N} .
$$

For $\varepsilon \rightarrow$ 0, Eq. (90) becomes the Roepstorff-Lieb inequality (64), which establishes the necessity of spontaneous gauge symmetry breaking for the BEC existence.

\section{Conservation of the number of particles}

If the spontaneous gauge symmetry breaking is a necessary condition for BEC, then where is a flaw in the standard argument of those who claim that BEC does not need gauge symmetry breaking and can be realized without the latter? This argument is as follows. When gauge symmetry has been broken, the number-of-particle operator $\hat{N}$ does not commute with the Hamiltonian $H_{\varepsilon}$, hence, $\hat{N}$ is not an integral of motion. However, in experiments, for instance with trapped atoms, the number of particles $N$ can be well controlled and kept constant during all the process of measurement.

\subsection{Conservation in the sense of quasiaverages}

The flaw in this argument is that it confuses the operator $\hat{N}$ with the observable quantity, which is the average $N=<\hat{N}>$. There is no any contradiction between the noncommutativity of an operator with a Hamiltonian, in which a symmetry is broken by infinitesimal sources, and the fact that the average of this operator can be well controlled and kept constant in experiments. The situation for BEC is analogous to that for magnetic systems. In the latter, the appearance of magnetization is accompanied by the spontaneous breaking of spin-rotational symmetry. When the latter is broken, the spin operator $\hat{\mathbf{S}}$ does not commute with the Hamiltonian. This, however, in no way forbids the magnetization, proportional to the spin average $<\hat{\mathbf{S}}>$, from being well controlled, accurately measured, and kept constant in experiments.

To demonstrate what is said above mathematically, let us consider the number-of-particle operator

$$
\hat{N} \equiv \int \psi^{\dagger}(\mathbf{r}) \psi(\mathbf{r}) d \mathbf{r}
$$

This operator commutes with the gauge-symmetric Hamiltonian $H[\psi]$,

$$
[H[\psi], \hat{N}]=0 .
$$

But after breaking the gauge symmetry with the infinitesimal source, the Hamiltonian $H_{\varepsilon}[\psi]$

in Eq. (27) does not commute with $\hat{N}$. Keeping in mind the separation of the field operator into two parts, as in Eq. (17), we have

$$
\left[\psi_{0}(\mathbf{r}), \hat{n}\left(\mathbf{r}^{\prime}\right)\right]=\varphi_{0}(\mathbf{r}) \varphi_{0}^{*}\left(\mathbf{r}^{\prime}\right) \psi\left(\mathbf{r}^{\prime}\right),
$$


where $\hat{n}(\mathbf{r}) \equiv \psi^{\dagger}(\mathbf{r}) \psi(\mathbf{r})$. From here,

$$
\left[\psi_{0}(\mathbf{r}), \hat{N}\right]=\psi_{0}(\mathbf{r})
$$

Therefore the commutator of $H_{\varepsilon} \equiv H_{\varepsilon}[\psi]$ with operator (91) is

$$
\left[H_{\varepsilon}, \hat{N}\right]=\varepsilon \sqrt{\rho} \int\left[\psi_{0}^{\dagger}(\mathbf{r})+\psi_{0}(\mathbf{r})\right] d \mathbf{r} .
$$

The number-of-particle operator (91) is now not an integral of motion, since the right-hand side of the Heisenberg equation

$$
i \frac{d}{d t} \hat{N}=\left[\hat{N}, H_{\varepsilon}\right]
$$

is not zero, being given by Eq. (93). But the observable quantities are the averages of operators. Hence, we should consider the averaged Eq. (94), which gives

$$
\frac{d}{d t}<\hat{N}>_{\varepsilon}=i<\left[H_{\varepsilon}, \hat{N}\right]>_{\varepsilon}
$$

In view of Eq. (93), we get

$$
\frac{d}{d t}<\hat{N}>_{\varepsilon}=i \varepsilon \sqrt{\rho} \int<\psi_{0}^{\dagger}(\mathbf{r})-\psi_{0}(\mathbf{r})>_{\varepsilon} d \mathbf{r}
$$

To correctly define thermodynamic limit, we have to work with reduced quantities. For example, Eq. (95) can be rewritten as

$$
\lim _{V \rightarrow \infty} \frac{d}{d t} \frac{<\hat{N}>_{\varepsilon}}{V}=i \lim _{V \rightarrow \infty} \frac{<\left[H_{\varepsilon}, \hat{N}\right]>_{\varepsilon}}{V}
$$

Using Eq. (93) and the Bogolubov theorem (51), we find

$$
\lim _{V \rightarrow \infty} \frac{d}{d t} \frac{<\hat{N}>_{\varepsilon}}{V}=i \varepsilon \lim _{V \rightarrow \infty} \frac{\sqrt{\rho}}{V} \int_{V}<\eta^{*}(\mathbf{r})-\eta(\mathbf{r})>_{\varepsilon} d \mathbf{r} .
$$

Breaking gauge symmetry by infinitesimal sources requires to define all observable quantities as Bogolubov quasiaverages. For the condensate function $\eta(\mathbf{r})$, rememebring normalization (41) and using the Cauchy-Schwarz inequality, we have

$$
\left|\int \eta(\mathbf{r}) d \mathbf{r}\right|^{2} \leq N_{0} V
$$

Because of this,

$$
\left|\frac{\sqrt{\rho}}{V} \int_{V}<\eta^{*}(\mathbf{r})-\eta(\mathbf{r})>_{\varepsilon} d \mathbf{r}\right| \leq 2 \sqrt{\rho \rho_{0}} .
$$

Therefore, from Eq. (98), we obtain

$$
\lim _{\varepsilon \rightarrow 0} \lim _{V \rightarrow \infty} \frac{d}{d t} \frac{<\hat{N}>_{\varepsilon}}{V}=0
$$


This means that the average number of particles $N$, defined by the relation

$$
\lim _{V \rightarrow \infty} \frac{N}{V}=\lim _{\varepsilon \rightarrow 0} \lim _{V \rightarrow \infty} \frac{<\hat{N}>_{\varepsilon}}{V},
$$

does not change in time and, being fixed at the initial time, can be kept constant during any experiment. The number of particles $N$, according to Eq. (100), can be thought as being defined by the asymptotic form $N \simeq<\hat{N}>_{\varepsilon}$, assuming that $\varepsilon \rightarrow 0$ and $N \rightarrow \infty$. Thus, though the operator $\hat{N}$ does not commute with $H_{\varepsilon}$, the observable number of particles $N$ is a well controlled quantity that can be fixed with any desired accuracy.

The form of Eq. (98) hints that the influence of the gauge-symmetry breaking source is in the appearance of a phase in $\langle\hat{N}\rangle_{\varepsilon}$, proportional to $\varepsilon$. This is easy to illustrate for a uniform system, when $\eta(\mathbf{r})=\sqrt{\rho_{0}}$. Then asymptotically, as $N \rightarrow \infty$, we have

$$
<\left[H_{\varepsilon}, \hat{N}\right]>_{\varepsilon} \sim 2 \varepsilon \sqrt{n_{0}} N,
$$

where

$$
n_{0} \equiv \lim _{N \rightarrow \infty} \frac{N_{0}}{N}
$$

is the condensate fraction. Keeping in mind that, in accordance with Eq. (100), asymptotically $N \simeq<\hat{N}>_{\varepsilon}$, we obtain from Eq. (98) the asymptotic equation

$$
\frac{d N}{d t} \sim 2 \varepsilon \sqrt{n_{0}} N .
$$

From here

$$
N(t) \sim N(0) \exp \left(2 \varepsilon \sqrt{n_{0}} t\right) .
$$

Passing again to quasiaverages, we get

$$
\lim _{\varepsilon \rightarrow 0} \lim _{V \rightarrow \infty} \frac{N(t)}{N(0)}=1 .
$$

This confirms once more that the observable number of particles can always be treated as a well defined, conserved, and precisely controlled quantity.

\subsection{Conservation under Bogolubov shift}

Moreover, the number-of-particle operator can be explicitly an integral of motion, at the same time with gauge symmetry being broken. This can be realized in the representation employing the Bogolubov shift. The initial Hamiltonian $H[\psi]$, which is a functional of the field operators $\psi$ and $\psi^{\dagger}$, is gauge invariant, according to property (26). Accomplishing the Bogolubov shift (34) leaves the operator structure of the new Hamiltonian $H[\hat{\psi}] \equiv$ $H\left[\eta, \psi_{1}\right]$ the same as that of $H[\psi]$. Hence, the Hamiltonian $H\left[\eta, \psi_{1}\right]$ is invariant under the transformation $\hat{\psi} \rightarrow \hat{\psi} e^{i \alpha}$, that is, $\eta \rightarrow \eta e^{i \alpha}$ and $\psi_{1} \rightarrow \psi_{1} e^{i \alpha}$. As far as $H[\psi]$, by assumption (26), is gauge invariant, it commutes with the number-of-particle operator $\hat{N}[\psi]$. Since the Bogolubov shift (34) does not change the operator structure, except for replacing $\psi$ by $\hat{\psi}$, the Hamiltonian $H\left[\eta, \psi_{1}\right]$ commutes with the number-of-particle operator

$$
\hat{N}\left[\eta, \psi_{1}\right] \equiv \int \hat{\psi}^{\dagger}(\mathbf{r}) \hat{\psi}(\mathbf{r}) d \mathbf{r},
$$


so that

$$
\left[\hat{N}\left[\eta, \psi_{1}\right], H\left[\eta, \psi_{1}\right]\right]=0
$$

Operator (106) can be written as the sum

$$
\hat{N}\left[\eta, \psi_{1}\right]=N_{0}+\hat{N}_{1}
$$

of the number of condensed particles (41) and the operator

$$
\hat{N}_{1} \equiv \int \psi_{1}^{\dagger}(\mathbf{r}) \psi_{1}(\mathbf{r}) d \mathbf{r}
$$

for the number of uncondensed particles. Thus the number-of-particle operator (106), in view of commutator (107), is the integral of motion, which means that the number of particles

$$
N \equiv<\hat{N}\left[\eta, \psi_{1}\right]>_{\eta}
$$

with the average defined in Eq. (37), is a conserved quantity, which can be measured and fixed with the desired accuracy. At the same time, the gauge symmetry, in compliance with Eq. (40), is broken.

In order to understand how it may happen that the number-of-particle operator is the integral of motion, but the gauge symmetry is broken, we should remember that symmetry can be broken not solely in the Hamiltonian, by adding infinitesimal sources, but also by constructing an operator representation on a space of microstates with broken symmetry [31]. To be more precise, we can invoke the decomposition theory [43-45].

For a finite system, the space of microstates is the Fock space $\mathcal{F}(\psi)$ generated by the field operator $\psi^{\dagger}$. The space is gauge invariant, in the sense that $\mathcal{F}\left(\psi e^{i \alpha}\right)=\mathcal{F}(\psi)$. The Fock space $\mathcal{F}\left(\psi_{1}\right)$, generated by $\psi_{1}^{\dagger}$, is not gauge invariant. Actually, one can define an infinite number of spaces

$$
\mathcal{F}_{\alpha} \equiv \mathcal{F}\left(\psi_{1} e^{i \alpha}\right) \quad(0 \leq \alpha<2 \pi)
$$

In thermodynamic limit, the sole space $\mathcal{F}(\psi)$ disintegrates into the direct integral

$$
\mathcal{F}=\int^{\oplus} \mathcal{F}_{a} \frac{d \alpha}{2 \pi}
$$

of the partial spaces (111).

On each of subspaces (111), one can define a representation of the operator algebra, whose members are denoted as

$$
\hat{A}_{\alpha} \equiv \hat{A}\left[\eta e^{i \alpha}, \psi_{1} e^{i \alpha}\right]
$$

For this representation, we can construct the corresponding statistical averages by introducing the statistical operator

$$
\hat{\rho}_{\alpha}(\eta) \equiv \frac{\exp \left(-\beta H_{\alpha}\right)}{\operatorname{Tr}_{\mathcal{F}_{\alpha}} \exp \left(-\beta H_{\alpha}\right)},
$$

in which $H_{\alpha} \equiv H\left[\eta e^{i \alpha}, \psi_{1} e^{i \alpha}\right]$, according to Eq. (113). Then we have

$$
<\hat{A}_{\alpha}>_{\eta \alpha} \equiv \operatorname{Tr}_{\mathcal{F}_{\alpha}} \hat{\rho}_{\alpha}(\eta) \hat{A}_{\alpha}
$$


It is easy to notice that

$$
\lim _{\alpha \rightarrow 0}<\hat{A}_{\alpha}>_{\eta \alpha}=<\hat{A}\left[\eta, \psi_{1}\right]>_{\eta},
$$

with the right-hand side given by Eq. (37). The average of an operator $\hat{A}$, defined on the gauge-invariant space (112), is decomposed into the integral

$$
<\hat{A}>_{\mathcal{F}}=\int_{0}^{2 \pi}<\hat{A}_{\alpha}>_{\eta \alpha} \frac{d \alpha}{2 \pi}
$$

over gauge-noninvariant averages defined for subspaces (111).

In conformity with condition (39), we require that

$$
<\psi_{1}(\mathbf{r})>_{\eta \alpha}=0 .
$$

Then the gauge-invariant average of $\hat{\psi}$ is

$$
<\hat{\psi}(\mathbf{r})>_{\mathcal{F}} \equiv \int_{0}^{2 \pi} \eta(\mathbf{r}) e^{i \alpha} \frac{d \alpha}{2 \pi}=0,
$$

as it should be. However, the average of the representation $\hat{\psi}_{\alpha}(\mathbf{r}) \equiv \hat{\psi}(\mathbf{r}) e^{i \alpha}$ over the gaugenoninvariant space (111) is not zero,

$$
<\hat{\psi}_{\alpha}(\mathbf{r})>_{\eta \alpha}=\eta(\mathbf{r}) e^{i \alpha},
$$

which implies gauge symmetry breaking. This is the way how the gauge symmetry of a system can be broken, despite that the number-of-particle operator remains the integral of motion.

One may also notice that, under the assumption that $\mathcal{F}(\psi)$ tends, in thermodynamic limit, to $\mathcal{F}$, given by decomposition (112), then

$$
\lim _{N \rightarrow \infty}<\psi_{0}^{\dagger}(\mathbf{r}) \psi_{0}(\mathbf{r})>=|\eta(\mathbf{r})|^{2} .
$$

This again proves that BEC is equivalent to gauge symmetry breaking. In other words, the latter is a necessary condition for the existence of BEC.

\section{$6 \quad$ Negligible condensate fluctuations}

In recent years much attention has been given to the study of condensate fluctuations (see review article [5]). One often claims that these fluctuations are anomalous or even catastrophic. However, if to calculate these fluctuations correctly, not forgetting about the broken gauge symmetry, which is a necessary condition, then they are neither anomalous nor catastrophic, but, moreover, they are negligible in thermodynamic limit.

One describes the condensate fluctuations as the fluctuations associated with the condensate number-of-particle operator

$$
\hat{N}_{0} \equiv \int \psi_{0}^{\dagger}(\mathbf{r}) \psi_{0}(\mathbf{r}) d \mathbf{r} .
$$

The fluctuations are characterized by the dispersion of operator (121),

$$
\Delta_{\varepsilon}^{2}\left(\hat{N}_{0}\right) \equiv<\hat{N}_{0}^{2}>_{\varepsilon}-<\hat{N}_{0}>_{\varepsilon}^{2} .
$$


The dispersion of an extensive operator is not, of course, an observable quantity by its own. The observable quantity is the ratio

$$
\lim _{N \rightarrow \infty} \frac{\Delta^{2}\left(\hat{N}_{0}\right)}{N} \equiv \lim _{\varepsilon \rightarrow 0} \lim _{N \rightarrow \infty} \frac{\Delta_{\varepsilon}^{2}\left(\hat{N}_{0}\right)}{N},
$$

which is analogous to the definition of other extensive observables [46,47]. Denoting

$$
\hat{n}_{0}(\mathbf{r}) \equiv \psi_{0}^{\dagger}(\mathbf{r}) \psi_{0}(\mathbf{r})
$$

we can write down dispersion (122) as

$$
\Delta_{\varepsilon}^{2}\left(\hat{N}_{0}\right)=\int\left[<\hat{n}_{0}(\mathbf{r}) \hat{n}_{0}\left(\mathbf{r}^{\prime}\right)>_{\varepsilon}-<\hat{n}_{0}(\mathbf{r})>_{\varepsilon}<\hat{n}_{0}\left(\mathbf{r}^{\prime}\right)>_{\varepsilon}\right] d \mathbf{r} d \mathbf{r}^{\prime}
$$

In thermodynamic limit, according to the Bogolubov theorem, we should make the replacement $\psi_{0}(\mathbf{r}) \rightarrow \eta(\mathbf{r})$, hence

$$
\hat{n}_{0}(\mathbf{r}) \rightarrow \rho_{0}(\mathbf{r}) \equiv|\eta(\mathbf{r})|^{2}
$$

Then Eq. (125) immediately gives $\Delta_{\varepsilon}^{2}\left(\hat{N}_{0}\right) \simeq 0$.

One may ask what happens if one represents dispersion (125) is a slightly different form following from the commutation relation (19)? Using the latter, we can write

$$
\hat{n}_{0}(\mathbf{r}) \hat{n}_{0}\left(\mathbf{r}^{\prime}\right)=\psi_{0}^{\dagger}(\mathbf{r}) \psi_{0}^{\dagger}\left(\mathbf{r}^{\prime}\right) \psi_{0}\left(\mathbf{r}^{\prime}\right) \psi_{0}(\mathbf{r})+\psi_{0}^{\dagger}(\mathbf{r}) \psi_{0}\left(\mathbf{r}^{\prime}\right) \varphi_{0}(\mathbf{r}) \varphi_{0}^{*}\left(\mathbf{r}^{\prime}\right)
$$

We should expect that the result for dispersion (125) could not depend on the used commutation relation (19), since the condensate operators $\psi_{0}(\mathbf{r})$ and $\psi_{0}^{\dagger}\left(\mathbf{r}^{\prime}\right)$, in agreement with Eq. (23), commute in thermodynamic limit. More accurately, there is the property

$$
\lim _{N \rightarrow \infty}<\left[\psi_{0}(\mathbf{r}), \psi_{0}^{\dagger}\left(\mathbf{r}^{\prime}\right)\right]>_{\varepsilon}=0
$$

One should exercise extreme caution integrating expressions containing the commutation relations (19). As is explained in Section 2, such expressions have to be understood in the sense of equations for distributions. Their integration is correctly defined only for the integration with an integrable function, as is specified in Section 2. Thus the integration of Eq. (127) over the spatial variables has sense only for the case

$$
\lim _{N \rightarrow \infty} \int_{V}<\left[\psi_{0}(\mathbf{r}), \psi_{0}^{\dagger}\left(\mathbf{r}^{\prime}\right)\right]>_{\varepsilon} f\left(\mathbf{r}, \mathbf{r}^{\prime}\right) d \mathbf{r} d \mathbf{r}^{\prime}=0
$$

where $f\left(\mathbf{r}, \mathbf{r}^{\prime}\right)$ is an integrable function, such as defined in subsection 2.2. The integration without such a function can yield wrong results. For example, we could get

$$
\int_{V}<\left[\psi_{0}(\mathbf{r}), \psi_{0}^{\dagger}\left(\mathbf{r}^{\prime}\right)\right]>_{\varepsilon} d \mathbf{r} \rightarrow 1
$$

for large $N \rightarrow \infty$, which would contradict the commutativity property (23). To exclude the appearance of such incorrect terms, one has to employ the regularized limit

$$
\lim _{N \rightarrow \infty} \frac{\Delta_{\varepsilon}^{2}(\hat{N})}{N}=
$$




$$
=\lim _{f \rightarrow 1} \lim _{N \rightarrow \infty} \frac{1}{N} \int_{V}\left[<\hat{n}_{0}(\mathbf{r}) \hat{n}_{0}\left(\mathbf{r}^{\prime}\right)>_{\varepsilon}-<\hat{n}_{0}(\mathbf{r})>_{\varepsilon}<\hat{n}_{0}\left(\mathbf{r}^{\prime}\right)>_{\varepsilon}\right] f\left(\mathbf{r}, \mathbf{r}^{\prime}\right) d \mathbf{r} d \mathbf{r}^{\prime} .
$$

Reducing the regularizing function $f\left(\mathbf{r}, \mathbf{r}^{\prime}\right)$ to unity can be done only after thermodynamic limit, but not before, since

$$
\left[\lim _{f \rightarrow 1}, \lim _{N \rightarrow \infty}\right] \neq 0 \text {. }
$$

Suppose, we decide to use form (126) in dispersion (125). Employing the equality $\psi_{0}(\mathbf{r})=$ $a_{0} \varphi_{0}(\mathbf{r})$, the Bogolubov replacement $a_{0} \rightarrow \sqrt{N_{0}}$, condition (22), and taking care of correct definitions, we have

$$
\begin{gathered}
\lim _{N \rightarrow \infty} \frac{1}{N}\left|\int_{V}<\psi_{0}^{\dagger}(\mathbf{r}) \psi_{0}\left(\mathbf{r}^{\prime}\right)>_{\varepsilon} \varphi_{0}(\mathbf{r}) \varphi_{0}^{*}\left(\mathbf{r}^{\prime}\right) f\left(\mathbf{r}, \mathbf{r}^{\prime}\right) d \mathbf{r} d \mathbf{r}^{\prime}\right| \leq \\
\leq \lim _{N \rightarrow \infty}\left|\rho_{0} \frac{\text { const }}{N^{2 \nu}} \int_{V} f\left(\mathbf{r}, \mathbf{r}^{\prime}\right) d \mathbf{r} d \mathbf{r}^{\prime}\right|=0 .
\end{gathered}
$$

Thence, the additional term in Eq. (126), caused by the commutation relations (19), does not contribute to the reduced dispersion (129). In any case, we have

$$
\lim _{N \rightarrow \infty} \frac{\Delta_{\varepsilon}^{2}\left(\hat{N}_{0}\right)}{N}=0
$$

This means that the condensate fluctuations are negligible in thermodynamic limit.

If we would employ representation (15) in the definition of operator (121), we would get

$$
\hat{N}_{0}=\int \hat{n}_{0}(\mathbf{r}) d \mathbf{r}=a_{0}^{\dagger} a_{0}
$$

Hence,

$$
\hat{N}_{0}^{2}=a_{0}^{\dagger} a_{0} a_{0}^{\dagger} a_{0} \text {. }
$$

Using here the commutation relation $\left[a_{0}, a_{0}^{\dagger}\right]=1$, we have another form

$$
\hat{N}_{0}^{2}=a_{0}^{\dagger} a_{0}^{\dagger} a_{0} a_{0}+a_{0}^{\dagger} a_{0}
$$

It looks like Eqs. (132) and (133) give different answers for dispersion (125). For instance, keeping in mind the Bogolubov replacement $a_{0} \rightarrow \sqrt{N_{0}}$ and using form (132), we have $\Delta_{\varepsilon}^{2}\left(\hat{N}_{0}\right)=0$, in agreement with Eq. (130). At the same time, using form (133), we would get $\Delta_{\varepsilon}^{2}\left(\hat{N}_{0}\right) \rightarrow N_{0}$, which contradicts Eq. (130). The cause of this contradiction is that the spatial integrations, in the process of passing from the spatial representation to Eq. (131), have been done without a regularization function. To correct this defect, one has either to return to the spatial representation and to proceed as has been explained above or to invoke the following argumentation.

The Bogolubov replacement $a_{0} \rightarrow \sqrt{N_{0}}$ implies that the operators $a_{0}$ and $a_{0}^{\dagger}$ should commute in thermodynamic limit. More correctly,

$$
\lim _{N \rightarrow \infty} \frac{<\left[a_{0}, a_{0}^{\dagger}\right]>_{\varepsilon}}{N_{0}}=0
$$


where $N_{0}=<\hat{N}_{0}>_{\varepsilon}$ tends to infinity proportionally to $N$, as $N \rightarrow \infty$. To correctly define $<\hat{N}_{0}^{2}>_{\varepsilon}$, one has to consider the reduced quantity $<\hat{N}_{0}^{2}>_{\varepsilon} / N_{0}^{2}$. Then, form (132) gives

$$
\lim _{N \rightarrow \infty} \frac{<\hat{N}_{0}^{2}>_{\varepsilon}}{N_{0}^{2}}=1
$$

Form (133) yields the same answer

$$
\lim _{N \rightarrow \infty} \frac{<\hat{N}_{0}^{2}>_{\varepsilon}}{N_{0}^{2}}=\lim _{N \rightarrow \infty}\left(1+\frac{1}{N_{0}}\right)=1 .
$$

In that sense, one always has

$$
<\hat{N}_{0}^{2}>_{\varepsilon} \simeq N_{0}^{2} \quad(N \rightarrow \infty)
$$

independently of the form involved. Thus one always returns to Eq. (130) telling us that the condensate fluctuations are thermodynamically negligible.

One could come to catastrophic condensate fluctuations only by means of incorrect calculations, assuming the existence of BEC but without breaking gauge symmetry. In the latter case, using the Wick theorem, one would get

$$
<\hat{N}_{0}^{2}>=<a_{0}^{\dagger} a_{0}>\left(2+<a_{0}^{\dagger} a_{0}>\right)
$$

from where

$$
<\hat{N}_{0}^{2}>-<\hat{N}_{0}>^{2}=N_{0}\left(1+N_{0}\right),
$$

with $N_{0} \equiv<a_{0}^{\dagger} a_{0}>\propto N$. As a result, one finds

$$
<\hat{N}_{0}^{2}>-<\hat{N}_{0}>^{2} \propto N^{2}
$$

This what one calls the catastrophic condensate fluctuations, since the normal dispersion must be proportional to $N$. One often blames the grand ensemble for this unreasonable behavior of the dispersion. However, as has been just explained, it is not the grand ensemble to blame, but this fictitious catastrophe is merely provoked by wrong calculations. The correct way of treating the problem results in thermodynamically negligible condensate fluctuations in the sense of Eq. (130). It is worth stressing that result (130) is general and is valid for arbitrary Bose systems, whether uniform or nonuniform and even whether they are equilibrium or nonequilibrium.

\section{Representative statistical ensembles}

The basic point, analyzed and explained in this paper, is that spontaneous gauge symmetry breaking is the necessary and sufficient condition for the existence of BEC. Although it is, probably, possible to calculate some particular quantities of a Bose-condensed system without explicit symmetry breaking, but, in general, such a way of calculation is not correct and can lead to wrong results. There are two methods of gauge symmetry breaking, by introducing into the Hamiltonian infinitesimal sources or by means of the Bogolubov operator shift. These methods are equivalent in thermodynamic limit. However, before the latter, the 
methods are not equivalent, since they involve different operator variables possessing different commutation relations. To formulate this difference in a precise form, it is convenient to invoke the notion of representative statistical ensembles [31,46,48-50].

A statistical ensemble is a pair $\{\mathcal{F}, \hat{\rho}\}$ of the space of microstates $\mathcal{F}$ and a statistical operator $\hat{\rho}$. The latter, for an equilibrium system, is given by the Gibbs form characterized by the appropriate Hamiltonian, and for a nonequilibrium system, $\hat{\rho}$ is described by the Liouville equation, whose dynamics is governed by the related Hamiltonian.

A representative statistical ensemble is an ensemble equipped with all additional conditions that are necessary for a unique description of the given physical system [31,46]. Additional conditions may include symmetry properties, normalization conditions, conservation conditions, and so on. Actually, only representative ensembles have sense.

Suppose, we are considering a Bose system in the frame of the field-operator technique. With a field operator $\psi$, the space of microstates is the Fock space $\mathcal{F}(\psi)$ generated by $\psi^{\dagger}$. The field operators, as is well known, do not conserve the number of particles. Therefore, working with the field-operator technique, it is necessary to use the grand canonical ensemble fixing the average number of particles $N=<\hat{N}>$. The statistical operator $\hat{\rho}$ is defined by a Hamiltonian $H[\psi]$ which is a functional of $\psi$. To emphasize this, we shall write $\hat{\rho}[H(\psi)]$. Let us denote by $\hat{H}[\psi]$ the energy operator. And let us define the statistical ensemble

$$
\{\mathcal{F}(\psi), \hat{\rho}(H[\psi])\}, \quad H[\psi] \equiv \hat{H}[\psi]-\mu \hat{N} .
$$

This is the standard grand canonical ensemble with the grand Hamiltonian $H[\psi]$, which is assumed to be gauge invariant. Ensemble (136) is representative for a system without BEC. But as soon as the latter appears, ensemble (136) is not anymore representative, since BEC necessarily requires gauge symmetry breaking. Accomplishing this by means of the method of infinitesimal sources, adding a gauge-symmetry breaking term $\hat{\Gamma}$, we have the statistical ensemble

$$
\left\{\mathcal{F}(\psi), \hat{\rho}\left(H_{\varepsilon}[\psi]\right)\right\}, \quad H_{\varepsilon}[\psi] \equiv \hat{H}[\psi]-\mu \hat{N}+\varepsilon \hat{\Gamma} .
$$

This ensemble is representative for a system with BEC, the operator averages being defined as the Bogolubov quasiaverages. The number of particles is now given by $N=\langle\hat{N}\rangle_{\varepsilon}$. The chemical potential $\mu$ is the Lagrange multiplier preserving the normalization condition for the number of particles $N$. In ensemble (137), there is the sole field variable $\psi$ and the only normalization condition for $N$. Hence, just one Lagrange multiplier is required. The representation (17) for $\psi$, as a sum of two terms, is rather formal, since neither of these terms comprises an independent variable. The parts of sum (17) are interconnected through their commutation relations (19), (20), and (21). It is, of course, possible to work with ensemble (137), invoking perturbation theory in powers of particle interactions, as has been done by Belyaev [35]. This, however, has two weak points. First, such a perturbative way makes it possible to deal exclusively with weakly interacting systems. Second, this way is very cumbersome because of the complicated commutation relations (19) and (20).

It is tempting to resort to the Bogolubov theorem and to make the Bogolubov shift (34), passing to the simple commutation relation (35). However, one has to remember that the Bogolubov shift becomes exact only in thermodynamic limit, but not before it. At the same time, all calculations are usually accomplished before thermodynamic limit. Using the commutation relations (35) before this limit is incorrect, makes the theory not selfconsistent, and leads to different paradoxes. The most dangerous such a paradox is the 
Hohenberg-Martin dilemma of conserving versus gapless theories [51]. This paradox has recently been resolved in Refs. [32,46,48-50,52,53].

The solution to the problem is merely the necessity of being mathematically accurate. Yes, we can employ the Bogolubov shift (34) and to use the simple commutation relations (35) even before thermodynamic limit, provided $N$ is large. But then it is necessary to understand that the Bogolubov shift introduces two independent variables, the condensate wave function $\eta(\mathbf{r})$ and the operator of uncondensed particles $\psi_{1}(\mathbf{r})$. So, there are now two normalization conditions, for the number of condensed particles

$$
N_{0}=\int|\eta(\mathbf{r})|^{2} d \mathbf{r}
$$

and for the number of uncondensed particles

$$
N_{1}=<\hat{N}_{1}>_{\eta}
$$

where $\hat{N}_{1}$ is given by Eq. (109) and the average is defined in Eq. (37). Preserving two normalization conditions requires two Lagrange multipliers. In addition, one must be aware of the fact that the Bogolubov shift (34) induces a new operator representation, which is nonequivalent to that used in ensemble (137). Now, the space of microstates is the Fock space $\mathcal{F}\left(\psi_{1}\right)$ generated by $\psi_{1}^{\dagger}$. The space $\mathcal{F}\left(\psi_{1}\right)$ is asymptotically orthogonal to the space $\mathcal{F}(\psi)$ of ensemble (137), with all operators forming the new representation on this space [32]. After accomplishing the Bogolubov shift, we come to the representative ensemble

$$
\left\{\mathcal{F}\left(\psi_{1}\right), \hat{\rho}\left(H\left[\eta, \psi_{1}\right]\right)\right\}, \quad H\left[\eta, \psi_{1}\right] \equiv \hat{H}\left[\eta, \psi_{1}\right]-\mu_{0} N_{0}-\mu_{1} \hat{N}_{1}
$$

with the statistical operator (38) and the grand Hamiltonian $H\left[\eta, \psi_{1}\right]$ which is a functional of two variables and which contains two Lagrange multipliers, $\mu_{0}$ and $\mu_{1}$, guaranteeing the validity of two normalization conditions (138) and (139). Since the Bogolubov shift explicitly breaks gauge symmetry, we do not need to invoke infinitesimal sources. The representative ensemble (140) is the basis for developing a completely self-consistent theory of Bose-condensed systems [46,48-50], which is free of paradoxes and which enables the description of strongly interacting systems with BEC $[52,53]$.

The case of Bose-condensed systems serves as a very illustrative example emphasizing the principal importance of defining a representative statistical ensemble for the system under consideration. It may happen that several ensembles can correctly describe a statistical system. Then one says that these ensembles are equivalent. For instance, the majority of statistical systems can be equivalently treated by means of either grand canonical, canonical, or microcanonical ensembles. But this is not compulsory. Even more, pronouncing just the term of an ensemble, say, grand canonical, is not sufficient, since, as has been shown above, there can exist several grand canonical ensembles. Thus ensembles (136), (137), and (140) all are grand canonical, though they are different and not all equivalent. The most important is that the employed ensemble be representative. In the other case, it is unable to correctly represent the considered system. Several ensembles are equivalent if and only if they are representative [46]. There are some models with long-range interactions for which the microcanonical and canonical ensembles are not equivalent [54,55]. But this in no way is a fact that would shake the basis of statistical mechanics. This just means that one or both of the used ensembles are not representative. Say, the microcanonical ensemble may 
occur to be not representative for such mean-field models. Or it may be that some additional constraints are to be imposed in order to correctly define the model and to make ensembles representative, hence, equivalent.

In conclusion, it is worth emphasizing that the principal problems analyzed in the present paper concern any kind of Bose systems with BEC, whether uniform or nonuniform. These could be uniform Bose gases and superfluid liquids or nonuniform clouds of trapped Bose atoms. As is mentioned in the Introduction, multiquark clusters can also form BEC in the interiors of neutrons stars and in fireballs created in heavy-ion collisions [17-19]. Another example is the BEC of excitons [56,57]. Nonuniform condensates can appear in a uniform system, when the spectrum of collective excitations $[58,59]$ or the single-particle spectrum [60-62] touch zero at the finite momentum $\mathbf{k}_{0}$. Then a condensate with a periodic structure, prescribed by the momentum $\mathbf{k}_{0}$, arises, forming a coherent crystal [58,59]. A similar periodic density evolves in nuclear matter under pion condensation [63-72]. Periodic condensates can occur, in general, for any Bose particles in external fields [71,73]. Finiteness of a sample also can impose boundary conditions that would induce a periodic BEC, such as happens in magnetic films under magnon condensation [74,75]. The abundance of different types of BEC makes crucial the necessity of understanding the basic features of its correct theoretical description.

Acknowledgement. I am grateful to E. Lieb for useful correspondence on the relation between BEC and gauge symmetry breaking. 


\section{References}

[1] L. Pitaevskii and S. Stringari, Bose-Einstein Condensation in Dilute Gases (Clarendon, Oxford, 2003).

[2] P.W. Courteille, V.S. Bagnato, and V.I. Yukalov, Laser Phys. 11, 659 (2001).

[3] J.O. Andersen, Rev. Mod. Phys. 76, 599 (2004).

[4] K. Bongs and K. Sengstock, Rep. Prog. Phys. 67, 907 (2004).

[5] V.I. Yukalov, Laser Phys. Lett. 1, 435 (2004).

[6] V.I. Yukalov and M.D. Girardeau, Laser Phys. Lett. 2, 375 (2005).

[7] D.G. Fried, T.C. Killian, L. Willmann, D. Landhuis, S.C. Moss, D. Kleppner, and T.J. Greytak, Phys. Rev. Lett. 81, 3811 (1998).

[8] T. Weber, J. Herbig, M. Mark, H.C. Nägerl, and R. Grimm, Science 299, 232 (2003).

[9] Y. Takasu, K. Maki, K. Komori, T. Takano, K. Honda, M. Kumakura, T. Yabuzaki, and Y. Takahashi, Phys. Rev. Lett. 91, 040404 (2003).

[10] J. Yin, Phys. Rep. 430, 1 (2006).

[11] P. Noziéres and D. Pines, Theory of Quantum Liquids (Addison-Wesley, Reading, 1989).

[12] G.V. Chester and L. Reatto, Phys. Rev. B 22, 5199 (1980).

[13] T.R. Sosnick, W.M. Snow, P.E. Sokol, and R.N. Silver, Europhys. Lett. 9, 707 (1989).

[14] T.R. Sosnick, W.M. Snow, and P.E. Sokol, Phys. Rev. B 41, 11185 (1990).

[15] F.W. Wirth, D.E. Ewen, and R.B. Hallock, Phys. Rev. B 27, 5530 (1983).

[16] F.W. Wirth and R.B. Hallock, Phys. Rev. B 35, 89 (1987).

[17] V.I. Yukalov and E.P. Yukalova, Phys. Part. Nucl. 28, 37 (1997).

[18] V.I. Yukalov and E.P. Yukalova, Physica A 243, 382 (1997).

[19] A. Faessler, A.J. Buchmann, and M.I. Krivoruchenko, Phys. Rev. C 57, 1458 (1998).

[20] E.J. Mueller, T.L. Ho, M. Ueda, and G. Baym, Phys. Rev. A 74, 033612 (2006).

[21] O. Penrose and L. Onsager, Phys. Rev. 104, 576 (1956).

[22] A.J. Coleman and V.I. Yukalov, Reduced Density Matrices (Springer, Berlin, 2000).

[23] V.I. Yukalov, Physica A 310, 413 (2002).

[24] V.I. Yukalov, Statistical Green's Functions (Queen's University, Kingston, 1998).

[25] V.I. Yukalov, Phys. Rev. A 72, 033608 (2005). 
[26] F. Dalfovo, S. Giorgini, L.P. Pitaevskii, and S. Stringari, Rev. Mod. Phys. 71, 463 (1999).

[27] N.N. Bogolubov, Lectures on Quantum Statistics (Gordon and Breach, New York, 1967), Vol. 1.

[28] N.N. Bogolubov, Lectures on Quantum Statistics (Gordon and Breach, New York, 1970), Vol. 2.

[29] V.I. Yukalov, Physica A 108, 402 (1981).

[30] V.I. Yukalov, Int. J. Mod. Phys. B 5, 3235 (1991).

[31] V.I. Yukalov, Phys. Rep. 208, 395 (1991).

[32] V.I. Yukalov, Laser Phys. 16, 511 (2006).

[33] N.N. Bogolubov, J. Phys. (Moscow) 11, 23 (1947).

[34] N.N. Bogolubov, Moscow Univ. Phys. Bull. 7, 43 (1947).

[35] S.T. Belyaev, J. Exp. Theor. Phys. 7, 289 (1958).

[36] J. Ginibre, Commun. Math. Phys. 8, 26 (1968).

[37] E.H. Lieb, R. Seiringer, J.P. Solovej, and J. Yngvason, The Mathematics of the Bose Gas and Its Condensation (Birkhauser, Basel, 2005).

[38] E.H. Lieb, R. Seiringer, and J. Yngvason, Phys. Rev. Lett. 94, 080401 (2005).

[39] E.H. Lieb, R. Seiringer, and J. Yngvason, math-ph/0610034 (2006).

[40] A.B. Kuklov and J.L. Birman, Phys. Rev. A 63, 013609 (2000).

[41] G. Roepstorff, J. Stat. Phys. 18, 191 (1978).

[42] R.B. Griffiths, Phys. Rev. 152, 240 (1966).

[43] J. Dixmier, Les C*-Algebras et Leurs Representations (Gauthier-Villars, Paris, 1969).

[44] G.G. Emch, Algebraic Methods in Statistical Mechanics and Quantum Field Theory (Wiley, New York, 1972).

[45] O. Bratteli and D.W. Robinson, Operator Algebras and Quantum Statistical Mechanics (Springer, New York, 1979).

[46] V.I. Yukalov, Phys. Rev. E 72, 066119 (2005).

[47] V.I. Yukalov, Phys. Lett. A 340, 369 (2005).

[48] V.I. Yukalov, Laser Phys. Lett. 3, 406 (2006).

[49] V.I. Yukalov, Phys. Lett. A 359, 712 (2006). 
[50] V.I. Yukalov, Int. J. Mod. Phys. B 21, 69 (2007).

[51] P.C. Hohenberg and P.C. Martin, Ann. Phys. 34, 291 (1965).

[52] V.I. Yukalov and H. Kleinert, Phys. Rev. A 73, 063612 (2006).

[53] V.I. Yukalov and E.P. Yukalova, Phys. Rev. A 74, 063623 (2006).

[54] R.S. Ellis, H. Touchette, and B. Turkington, Physica A 335, 518 (2004).

[55] M. Costenine, R.S. Ellis, H. Touchette, and B. Turkington, Phys. Rev. E 73, 026105 (2006).

[56] D.W. Snoke, J.P. Wolfe, and A. Myzyrowicz, Phys. Rev. B 41, 11171 (1990).

[57] B. Link and G. Baym, Phys. Rev. Lett. 69, 2959 (1992).

[58] D.A. Kirzhnitz and Y.A. Nepomnyashchy, J. Exp. Theor. Phys. 59, 2203 (1970).

[59] Y.A. Nepomnyashchy and A.A. Nepomnyashchy, Theor. Math. Phys. 9, 137 (1971).

[60] V.I. Yukalov, Theor. Math. Phys. 27, 1093 (1978).

[61] V.I. Yukalov, Physica A 100, 431 (1980).

[62] V.I. Yukalov, Physica B 107, 233 (1981).

[63] A. B. Migdal, J. Exp. Theor. Phys. 61, 2209 (1971).

[64] R. Dashen and J.T. Manassah, Phys. Lett. B 50, 460 (1974).

[65] R. Dashen and J.T. Manassah, Phys. Lett. A 47, 453 (1974).

[66] D. Campbell, R. Dashen, and J.T. Manassah, Phys. Rev. D 12, 979 (1975).

[67] D. Campbell, R. Dashen, and J.T. Manassah, Phys. Rev. D 12, 1010 (1975).

[68] G. Baym, D. Campbell, R. Dashen, and J.T. Manassah, Phys. Lett. B 58, 304 (1975).

[69] D. Campbell, R. Dashen, and J.T. Manassah, Phys. Rev. D 18, 1343 (1978).

[70] A.B. Migdal, Rev. Mod. Phys. 50, 107 (1978).

[71] A.B. Migdal, Fermions and Bosons in Strong Fields (Nauka, Moscow, 1978).

[72] A.B. Migdal, D.N. Voskresensky, E.E. Saperstein, and M.A. Troitsky, Pion Degrees of Freedom in Nuclear Matter (Nauka, Moscow, 1991).

[73] V.I. Yukalov and A.S. Shumovsky, Lectures on Phase Transitions (World Scientific, Singapore, 1990).

[74] G.E. Volovik, cond-mat/0701180 (2007).

[75] A.I. Bugrij and V.M. Loktev, cond-mat/0701437 (2007). 